\title{
A morfossintaxe do aspecto em línguas Jê: uma abordagem diacronica
}

\author{
The morphosyntax of aspect in Jê languages: a diachronic \\ approach \\ Maxwell Miranda ${ }^{1}$
}

Recebido em outubro/2019 e aceito em outubro/2019.

\section{Resumo}

Este artigo examina comparativamente a morfossintaxe de construções oracionais que empregam verbos auxiliares para expressão de aspecto em línguas Jê meridionais e setentrionais, com vistas à reconstrução sintática de um antigo padrão sintático original a partir do qual os padrões encontrados na atualidade nas línguas desses dois ramos da família se desenvolveram. O presente estudo explora a constituição morfossintática das orações com verbos auxiliares, bem como destaca os principais verbos auxiliares usados na codificação de aspecto nas respectivas línguas. A análise fundamenta-se na Teoria da Gramaticalização (Heine 1993, 2018; Heine e Kuteva 2007), cuja metodologia de reconstrução repousa em três componentes principais: (a) reconstrução diacrônica via método comparativo, (b) reconstrução interna, e (c) generalização tipológica. Os resultados indicam que a variação sincrônica encontrada nas línguas investigadas é o reflexo de mudanças diacrônicas contínuas e graduais que levaram ao desenvolvimento de construções oracionais com verbos auxiliares, os quais têm se tornado cada vez mais gramaticalizados, principalmente, nas línguas Jê meridionais. Assim, a análise comparativa demonstra a regularidade das construções oracionais com verbos auxiliares, cujas formas são reconstruídas para o ProtoJê (Davis 1966, 1968), tornado possível a reconstrução do padrão sintático original a partir do qual se desenvolveram as construções com verbos auxiliares em boa parte das línguas da família.

Palavras-chave: Verbos auxiliares. Aspecto. Morfossintaxe. Línguas Jê. Tronco Macro-Jê.

\footnotetext{
Abstract

This article examines comparatively the morphosyntax of clause constructions that employ auxiliary verbs for the expression of aspect in southern and northern Jê languages in order

1 Professor Adjunto II da Universidade Federal de Mato Grosso (UFMT), Campus Universitário do

Araguaia (CUA), em Barra do Garças - MT, e do Programa de Pós-Graduação em Estudos de Linguagem

(PPGEL/UFMT).
} 
to reconstruct an original syntactic pattern from which the patterns found currently in the languages of these two branches of the family have developed. The study explores the morphosyntactic constitution of clauses with auxiliary verbs, as well as the main auxiliary verbs used in coding aspect. The analysis is based on the theory of grammaticalization (Heine 1993, 2018; Heine and Kuteva 2007), whose methodology rests on three main components: (a) diachronic reconstruction via comparative method, (b) internal reconstruction, and (c) typological generalization. The results show that the synchronic variation found in the investigated languages are the reflects of continuous and gradual diachronic changes that led to the development of clause constructions with auxiliary verbs, some of which have become increasingly grammaticalized, especially in the Southern Jê languages. The comparative analysis demonstrates the regularity of close constructions with auxiliary verbs, whose forms are reconstructed for Proto-Jê (Davis 1966, 1968), making it possible to reconstruct the original syntactic pattern from which the constructions with auxiliary verbs were developed in most languages of the Jê family.

Keywords: Auxiliary Verbs. Aspect. Morphosyntax. Diachrony. Languages Jê. Macro-Jê stock.

\section{Introdução}

Construções oracionais com verbos auxiliares são um dos traços morfossintáticos mais notáveis de grande parte das línguas da família Jê (Macro-Jê), e podem ser usadas para diferentes funções em diversos contextos morfossintáticos. Embora seja nítido o papel que tais orações desempenham na gramática dessas línguas, ainda não foram submetidas à uma análise comparativa, a fim de estabelecer correspondências sistemáticas de forma e função com vistas à reconstrução sintática de padrões oracionais relativos a diferentes estágios históricos da família. Uma análise dessa natureza pode auxiliar-nos a estabelecer e prever possíveis direções de mudança linguística, as quais resultaram em graus variados de gramaticalização dentro da família Jê nesse domínio da gramática.

O objetivo deste artigo é examinar a expressão morfossintática do aspecto mediante o uso de verbos auxiliares em diversas línguas Jê, e demonstrar a regularidade dessas construções dentro da família, tornando possível a reconstrução sintática do padrão oracional a partir do qual as orações atuais se desenvolveram nas línguas dos ramos setentrional e meridional. Nessa perspectiva, argumento que os casos existentes de variação sincrônica são o reflexo de diferentes estágios de gramaticalização decorrentes de mudanças diacrônicas contínuas e graduais.

$\mathrm{O}$ presente artigo $^{2}$ organiza-se nas seguintes seções. Na seção Verbos

2Agradeço ao povo krahô, da comunidade Pedra Branca, com qual tenho convivido e aprendido um pouco de sua língua e cultura e, em especial, às minhas pĩxwỳj, Letícia Jõhkàhkwy”j Krahô e Creuza Prũmkwỳj; meus ikwỳnõ (amigos), Edivaldo Wakê Krahô, Lindalva Craxỳ Krahô, Potyt Krahô, José Miguel (Cõc) Krahô e Pajhôt Krahô;

74 Revista Brasileira de Linguística Antropológica 
auxiliares: uma caracterização funcional, apresento uma caracterização funcional dos verbos auxiliares nas línguas Jê e mostro os diferentes papéis que podem desempenhar no desenvolvimento de novas funções gramaticais, como expressões de tempo, modo e aspecto, seguindo Heine (1993). A seção intitulada Propriedades morfossintáticas das orações com verbos auxiliares trata das propriedades morfossintáticas das orações com verbos auxiliares em línguas Jê e as respectivas categorias aspectuais às quais esses verbos se associam. $\mathrm{Na}$ sequência, a seção Uma proposta de reconstrução da morfossintaxe de aspecto na família é destinada à reconstrução sintática de um padrão oracional existente em um estágio anterior das línguas investigadas aqui, com base na metodologia proposta por (Heine 2018). Destaco, ainda, nessa seção, as possíveis direções de mudança linguística que levaram distintos cenários de gramaticalização. Na última seção, sublinho os principais resultados alcançados com o presente estudo e suas contribuições para futuras pesquisas acerca do papel que os verbos auxiliares desempenham na família Jê, assim como da importância da reconstrução sintática de aspectos gramaticais da família como um todo.

\section{Verbos auxiliares: uma caracterização funcional}

Verbos auxiliares, em diferentes línguas do mundo, podem apresentar uma multiplicidade de formas e funções nas construções oracionais das quais fazem parte. Esses verbos são compreendidos como combinações monoracionais de forma e função, que ocorrem em diversos tipos de construções e são usados para expressar categorias de tempo, modo e aspecto (Anderson 2006; Heine 1993). Payne (1998:84), além de destacar as funções semânticas típicas envolvidas no uso de verbos auxiliares, destaca também propriedades morfológicas e sintáticas comumente associadas à classe de palavras à qual eles se vinculam, isto é, a dos verbos, como por exemplo, a expressão formal de algum tipo de informação flexional, concordância sujeito/objeto e marcação de tempo/modo/aspecto, e a ocorrência em posições específicas na sentença.

Verbos auxiliares denotam primariamente eventos e estados, e abrangem conceitos básicos, como atividades, processos, localização, movimento, volição, posição/postura, relação e posse (Harris e Ramat 1987). A partir desses conceitos básicos, Heine (1993:31) propõe os seguintes esquemas principais como prováveis fontes para a gramaticalização de categorias gramaticais de tempo e aspecto (Tabela 1).

a minha família krahô, Hacàc Krahô, Xôhtyc Krahô, Pêt Krahô (itõ), Ceci Krahô e ao meu hõpin (amigo formal), Domingos Crate. Agradeço também aos meus amigos Tempty Suyá, Yaconhongarti Suya e Nanblá Gakran, os quais têm colaborado e me auxiliado a compreender certos fatos gramaticais de línguas Jê. É desnecessário dizer que eventuais erros e/ou equívocos são de minha inteira responsabilidade. 
Tabela 1. Principais esquemas de eventos (Heine 1993:31)

\begin{tabular}{ll}
\multicolumn{1}{c}{ Forma Conceitual } & \multicolumn{1}{c}{ Rótulo Proposto } \\
\hline a. "X está em Y" & Local \\
b. "X move para/a partir de Y" & Movimento \\
c. "X faz Y" & Ação \\
d. "X quer Y" & Volição \\
e. "X torna-se Y" & Mudança de estado \\
f. "X é (igual) um Y" & Igualdade \\
h. "X está com Y" & Companhia \\
g. "X tem Y" & Posse \\
h. "X fica em um modo Y" & Modo \\
\hline
\end{tabular}

As funções gramaticais mais comuns derivadas a partir desses esquemas abrangem diferentes contrastes temporais e aspectuais, como os que são apresentados na Tabela 2 (Heine 1993:47).

Tabela 2. Esquemas de evento e funções gramaticais

$\begin{array}{ll}\text { Local } & \text { Progressivo, ingressivo, contínuo } \\ \text { Movimento } & \text { Ingressivo, futuro, perfeito, passado } \\ \text { Ação } & \text { Progressivo, contínuo, ingressivo, completivo, perfeito } \\ \text { Volição } & \text { Ingressivo, futuro } \\ \text { Mudança de estado } & \text { Ingressivo, futuro } \\ \text { Igualdade } & \text { Resultativo, progressivo, perfeito, futuro } \\ \text { Companhia } & \text { Progressivo } \\ \text { Posse } & \text { Resultativo, perfeito, futuro } \\ \text { Modo } & \text { Progressivo }\end{array}$

Diversas línguas da família Jê apresentam um conjunto consistente e limitado de verbos que funcionam como auxiliares, usados para contrastar diferentes valores aspectuais (Miranda, no prelo). Cabral et al. (2018) mostram que parte desses verbos compartilha formas cognatas que expressam as mesmas categorias aspectuais, mas exprimindo valores distintos. As correspondências formais e funcionais indicam, por um lado, a presença de verbos auxiliares em estágios históricos mais antigos da família e, por outro, que as construções oracionais nas quais eles figuram nas línguas modernas podem ser provenientes de uma mesma fonte diacrônica. Assim, variação sincrônica encontrada na expressão morfossintática de aspecto pode ser explicada com base nas mudanças gramaticais contínuas que teriam sido impulsionadas pelo desmembramento e consequente diversificação das línguas da família.

As línguas Jê que dispõem de verbos auxiliares ${ }^{3}$ empregam comumente

3As únicas exceções dentro da família são as línguas do ramo central, Xerente e Xavante, e Panará, do ramo setentrional.

76 Revista Brasileira de Linguística Antropológica 
os esquemas (b), (c) e (h) (Tabela 1) e exprimem valores aspectuais como progressivo, continuativo, habitual, ingressivo e terminativo, cuja distribuição apresento no Quadro 1, com suas respectivas formas verbais plenas e nominalizadas (não-finitas), singulares/plurais e duais ${ }^{4}$.

\section{Quadro 1: Verbos auxiliares em línguas Jê}

\begin{tabular}{|c|c|c|c|}
\hline Perfectivo & \multicolumn{3}{|c|}{$\begin{array}{l}\text { 'completivo': Kra -pa(-r); Kdj-hwa (> matar.PL); } \\
\text { 'acabar': Kra -amrẽ (-r); } \\
\text { 'terminar': Apj-apeč } \\
\text { 'ir.PL': Kng/Lak - mũ }\end{array}$} \\
\hline \multirow[t]{3}{*}{ Imperfectivo } & Habitual & \multicolumn{2}{|c|}{ 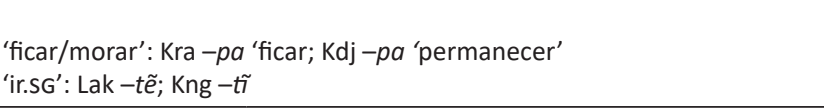 } \\
\hline & \multirow[t]{2}{*}{ Contínuo } & Não progressivo & $\begin{array}{l}\text { 'continuar': Kra -kre; } \\
\text { 'ir': Lak -tẽ ; Kra -tẽe(-m); } \\
\text { 'estativo': Lak -wã. } \\
\text { 'parar': Kra-ikuw }\end{array}$ \\
\hline & & Progressivo & 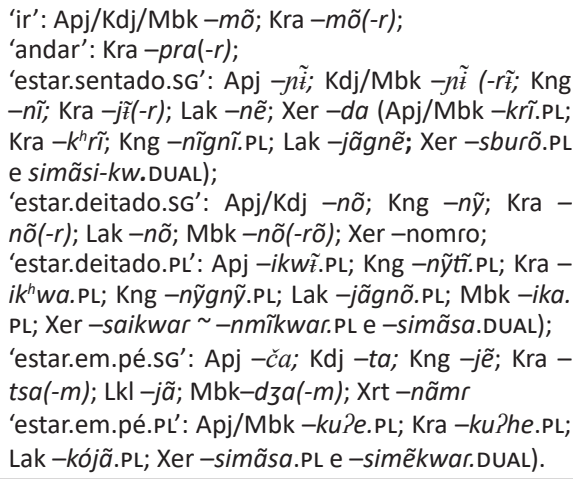 \\
\hline
\end{tabular}

Como pode ser notado, no Quadro 1, a maior parte dos esquemas de evento com suas respectivas funções gramaticais envolve os mesmos verbos auxiliares, embora seu valor aspectual possa diferir em maior ou menor grau, como é o caso do verbo de movimento $-m \tilde{o}(-r)$, que nas línguas Jê setentrionais expressa aspecto progressivo (imperfectivo), enquanto nas línguas do ramo meridional - Kaingáng e Laklãnõ (Xokléng) - o verbo -mũ exprime aspecto perfectivo (Gonçalves 2007, 2011; Gakran 2015). Maiores detalhes sobre as propriedades morfossintáticas das orações com verbos auxiliares serão discutidos mais adiante.

Outro ponto a ser destacado diz respeito à seleção do verbo auxiliar. Em línguas Jê setentrionais, o aspecto progressivo pode ser marcado tanto pelo verbo $-m \tilde{o}(-r)$ 'ir' quanto por um dos verbos posicionais. Desse modo, além do valor aspectual que se pretende transmitir na sentença, a escolha do verbo

4Abreviatura das línguas: APJ: Apinajé; LAK: Laklãnõ (Xokleng); KDJ: Kĩsêdjê (Suyá); KNG: Kaingang; KRA: Krahô; MBK: Mẽbêngôkre; XRT: Xerente. 
auxiliar depende das experiências cognitivas dos falantes acerca de eventos/ estados tipicamente denotadas por esses verbos. Essas escolhas envolvem principalmente verbos posicionais/posturais ${ }^{5}$, em que a posição e/ou localização que os referentes podem estar e/ou ocupar é determinante, bem como as atividades que são comumente realizadas em determinadas posições (Newman 2002; Watkins 1976).

$\mathrm{Na}$ seção seguinte, analiso as propriedades morfossintáticas das orações com verbos auxiliares. As línguas investigadas foram subdivididas em dois grupos principais (Grupo I e Grupo II), os quais correspondem respectivamente aos ramos setentrional e meridional, com base em propriedades gramaticais compartilhadas entre as línguas.

Propriedades morfossintáticas das orações com verbos auxiliares

A morfossintaxe das orações com verbos auxiliares na maioria das línguas Jê é relativamente consistente e regular com respeito à disposição dos constituintes na sentença, cuja ordem é SOV (Rodrigues 1999), na maioria das línguas Jê. As línguas que adotam essas orações como principal estratégia gramatical para expressão de aspecto apresentam com frequência o seguinte padrão sintático em (1):

$$
\text { SUJEITO [(OBJ. INDIR) (OBJ DIR) + NÚCLEO LEXICAL (POSP)] + AUX }
$$

Com base nesse padrão, podemos dividir as línguas em dois grupos principais, sendo o Grupo I formado pelas línguas que empregam posposições das quais os núcleos lexicais, isto é, nomes de ação, são complemento, e o Grupo II, por oposição, por aquelas que não empregam posposições nessa estrutura morfossintática. A presença de posposição desencadeia (a) uma série de reajustes morfossintáticos, como a nominalização do núcleo lexical, contribuindo, assim, para a distinção de valores aspectuais, e (b) o sistema de marcação de caso, cujos argumentos pronominais seguem o padrão NominativoAbsolutivo (Cabral e Costa 2004 [2002]) ${ }^{6}$.

\subsection{Línguas do Grupo I}

Em Apinajé, Mẽbêngôkre, Kĩsêdjê (Suyá) e Krahô (Timbira), orações no aspecto progressivo têm como auxiliar o verbo - mõ ou um dos verbos posicionais (cf. Quadro 1) e apresentam uma estrutura proposicional do tipo

5Usos especializados de verbos posicionais, para fins classificatórios, por exemplo, são encontrados em Laklãnõ (Xokléng), em que a sua escolha, além da posição (horizontal/ vertical), também é determinada por traços semânticos como forma, tamanho e consistência do referente nominal (Gakrán, comunicação pessoal).

6Esse padrão se caracteriza por marcar o sujeito intransitivo duplamente, tanto por pronomes nominativos quanto absolutivos, sendo que estes se cliticizam ao verbo. Nesse contexto estrutural, $\mathrm{S}$ e $\mathrm{O}$ são indexados pelos mesmos pronomes pessoais, enquanto o sujeito de núcleos lexicais transitivos não é marcado e é expresso apenas por pronomes nominativos.

78 Revista Brasileira de Linguística Antropológica 
$X$ ir/estar sentado/em példeitado com $Y$, conforme o esquema Comitativo (Tabela 2). Nessas línguas ${ }^{7}$, o núcleo lexical é determinado pela posposição instrumental to/ro/s e o núcleo lexical é um nome de ação derivado por meio de nominalização (Comrie 1976). Em diversas línguas Jê, a nominalização de temas verbais resulta na derivação de nomes de ação, os quais são formados por meio do sufixo nominalizador $-r(V)$ e seus alomorfes $-m,-n,-t,-k,-j$ ou $-\varnothing$ (Costa 2003; Miranda 2010; Gakran 2015; Cotrim 2016).

\section{Apinajé}

(1)

$\begin{array}{llllll}n a & p a & r & i c-t \dot{k} & 0 & m \tilde{o} \\ \text { RLS } & 1 & \text { ASP } & \text { 1-die.NF } & \text { do } & \text { go.AUX }\end{array}$

'I'm dying.' (Oliveira 2005:293)

(2) $k a \quad n a \quad k a \quad a$-grer $\quad \supset \quad c a$

2 RLS 2 2-sing.NF do stand.AUX

'You are dancing.' (Oliveira 2005:294)

\section{Mẽbêngôkres}

$\begin{array}{lllll}\text { Bir } & n \tilde{e} & k r \tilde{e}-n & \jmath & m \tilde{o} \\ \text { N.PESS } & \text { RLS } & \text { comer-NMLZ } & \text { INSTR } & \text { ir.AUX }\end{array}$

'Bir está comendo (em movimento)' (Stout e Thomson 1974:3)
$m \tilde{e}=k u n \tilde{\imath}$
na $m \tilde{e}=t \partial-r)$
$\varnothing-\supset$
d3a
$\mathrm{PL}=$ todos $\quad \mathrm{RLS} \quad \mathrm{PL}=$ festejar-NMLZ $\mathrm{R}^{1}$-ASS.INSTR
estar.em.pé.AUX

'Todos estão festejando.' (Costa 2015:305)

\footnotetext{
7Abreviaturas: $1=1^{\mathrm{a}}$ Pessoa; $2=2^{\mathrm{a}}$ Pessoa; ADVT $=$ Advertivo; ASP $=$ Apecto; ASSOC/ INSTR $=$ Associativo/Instrumental (Posposição); ATEN = Atenuativo; AUX = Auxiliar; CNJ $=$ Conjunção; CAUS $=$ Causativo , COMPL $=$ Completivo $;$ DAT $=$ Dativo; DIR $=$ Direcional; DUAL $=$ Dual $($ Número $) ;$ ENF $=$ Enfático; FEM $=$ Feminino; FOC $=$ Foco; $\mathrm{HAB}=$ Habitual; LOC =Locativo; IMPERF = Imperfectivo; INT = Interrogativo; IRLS = Irrealis; MS = Marca de Sujeito; MASC = Masculino; NF = Não-Finito; N.PESS = Nome pessoal; NEG = Negação; NOMLZ $=$ Nominalizador; OBL $=$ Oblíquo; RLS $=$ Realis; PERF $/$ PFV $=$ Perfectivo; POSP $=$ Posposição; PROG $=$ Progressivo; $\mathrm{PL}=$ Plural; $\mathrm{RL}=$ Relational Prefix; $\mathrm{R}^{1}=$ Relacional de Contiguidade; $\mathrm{R}^{2}=$ Relacional de não-contiguidade; REFLX $=$ Reflexivo; $\mathrm{RLS}=$ Realis; SING $=$ Singular; TRANS $=$ Translativo.

8Os dados da língua Mẽbêngôkre são provenientes das variedades faladas pelos povos Txukuhamẽi, Gorotire, Mẽkrãgnôti, publicados por Stout e Thomson (1975), e da língua Xikrín (Costa 2015).
} 


\section{Kĩsêdjê (Suyá) 9}
$h n$ $w a$
adzi-mbə-ro
ro
$h \tilde{t}$
ASP 1PL 1PL-chorar-NMLZ POSP
estar.sentado.AUX

'Nós estamos chorando' (Santos 1997:87)

(6)
$m m b i-y e$
$r a$
s-shwe-n
ro
ta
homem-PL MS
$\mathrm{R}^{2}$-trabalhar/fazer-NMLZ
POSP
em.pé.Aux
'Os homens estão trabalhando' (Santos 1997: 87)

\section{Krahô (Timbira)}

$p \varepsilon a=m \tilde{a}$ Pitwritre $\quad$-kato-r $\quad n \tilde{e} \quad i-k^{h} \boldsymbol{B}-\boldsymbol{\emptyset} \quad$-to $\quad m \tilde{o}$ então Lua $\mathrm{R}^{1}$-sair-NMLZ $\quad \mathrm{CNJ} \quad \mathrm{R}^{2}$-gritar-NMLZ $\mathrm{R}^{1}$-INSTR ir.AUX 'Então, Lua saiu e foi gritando.'(lit. Então, Lua houve o cantar de Lua e ele foi com o gritar dele) (Melatti 2010:53)
Hapor $\quad h-$-spe- $n$
$\varnothing-$ to
tsa
N.PESS $\quad \mathrm{R}^{2}$-comer-NMLZ $\mathrm{R}^{1}$-INSTR
estar.em.pé.AUX

'Hapôr está comendo (algo)

(Hapôr está em pé com o comer (de algo)) (Melatti 2010:53)
pije
ko ma
$m \tilde{e}=i n-k^{h} r \varepsilon-r$
$-t 5$
pra
mulher.PL rio DIR $\mathrm{PL}^{=} \mathrm{R}^{2}$-cantar-NOMLZ $\mathrm{R}^{1}$-INSTR andar.AUX 'As mulheres andam cantando na direção do rio.'
(lit. As mulheres andam com o cantar delas na direção do rio) (Melatti 2010:53)

Em Krahô, construções no aspecto progressivo podem também ser marcadas pelo verbo de movimento - pra (-r) 'andar'10, como mostra o exemplo (9). Conforme mencionado na nota 5, somente o sujeito de núcleos lexicais intransitivos é marcado duplamente (exemplos (1) e (2) do Apinajé e exemplo (5) do Kĩsêdjê (Suyá)). Argumentos nominais, entretanto, não são marcados por meio pronomes (exemplo (3) do Mẽbêngôkre, exemplo (6) do Kĩsêdjê (Suyá), e exemplos (7-9) do Krahô), sendo o núcleo lexical flexionado por prefixos
9 Os dados da língua Kĩsêdjê (Suyá) foram reanalisados por mim, a fim de oferecer uma exposição mais clara e abrangente dos argumentos apresentados aqui.
10 Em Kĩsêdjê (Suyá), é provável que a forma correspondente desse verbo seja mbra e também desempenhe a função de auxiliar, mas com valor habitual, e poderia ser traduzido no exemplo seguinte por "Liana anda alegre".
(a) liana $\mathrm{ra}$ kĩn kãm na mbra
N.PESS MS alegre POSP ? verbo.POS (SING)
'Liana é alegre.' (Santos 1997:89)

80 Revista Brasileira de Linguística Antropológica 
relacionais de não contiguidade. Já os núcleos lexicais transitivos compartilham o mesmo sujeito com o verbo auxiliar, conforme ilustram o exemplo (10) do Krahô e (11) do Kĩsêdjê (Suyá):
$i=\varnothing$-tõji $[\varnothing i$
$k^{h} w a r$
j-ate- $n$
$\varnothing$ Ø-to] $\tilde{j i i}$

$1 \mathrm{SG}=\mathrm{R}^{1}$-irmã mandioca $\mathrm{R}^{1}$-espremer-NMLZ $\mathrm{R}^{1}$-INST estar.sentado.AUX

'Minha irmã está sentada espremendo mandioca.'

(Minha esposa está sentada com o espremer da mandioca) (Miranda, notas de campo)

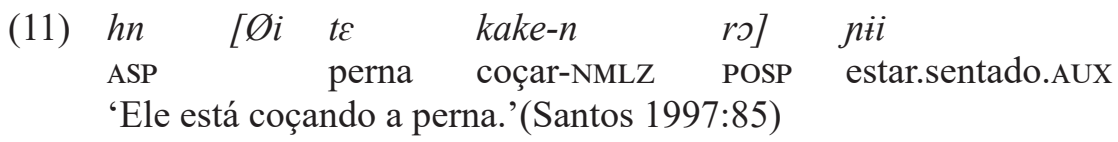

Construções oracionais no aspecto ingressivo parecem ser menos frequentes que aquelas no aspecto progressivo, têm como auxiliar o verbo de movimento -tẽe(-m) 'ir' e o núcleo lexical é complemento da posposição locativa $-k^{h} \tilde{a} m$ (exemplos (12) e (13) da língua Krahô (Miranda, notas de campo).
$\begin{array}{lllll}\text { wa } & h a & i=j \text { jpe- } n & \varnothing-k^{h} \tilde{a} m & t \tilde{e} \\ \text { 1SG } & \text { IRLS } & 1 \mathrm{SG}=\mathrm{R}^{1} \text {-trabalhar-NMLZ } & \mathrm{R}^{1} \text {-LOC } & \text { ir.AUX }\end{array}$
'Eu vou começar a trabalhar.' (lit. Eu vou em meu trabalho)
(13) $k u \quad$ ha põhi $\varnothing$-kajpre- $\varnothing \quad \varnothing-k^{h} \tilde{a} m \quad m \tilde{e}=t \tilde{e}$
1.DUAL IRLS milho $\mathrm{R}^{1}$-amarrar-NMLZ $\quad \mathrm{R}^{1}$-LOC $\quad \mathrm{PL}=$ ir.AUX
'Nós vamos começar a amarrar o milho'
(lit. Nós vamos na amarração do milho)

O aspecto continuativo, por sua vez, é expresso por meio da construção oracional que tem o verbo - $k r e$ 'continuar' como núcleo sintático, enquanto o núcleo lexical é complemento da posposição locativa $-k^{h} \tilde{a} m$ 'locativo' (exemplos (14) e (15)). Esse padrão oracional é encontrado apenas nas variedades Timbira - Canela-Ramkôkamekra ${ }^{11}$ e Krahô, como é mostrado nos exemplos (14) e (15).

11 Em Canela-Ramkôkamekra, ao contrário do Krahô, o complemento do verbo auxiliar pode ser determinado tanto pela posposição $k^{h} \tilde{a} m$ 'locativo' quanto to 'instrumental', tal como é indicado seguintes exemplos extraídos de Popjes e Popjes (1986:181). A variação quanto à posposição empregada, contudo, parece estar relacionada com a natureza semântica (evento vs. estado) do complemento do sintagma posposicional.

a-cakôc to a-crà

2-speak SUBORD 2-continue

'You continue to speak.'

$\begin{array}{llllll}\text { capi } & \text { te } & \text { rop } & k i ̃ & k a ̃ m & \text { incrà } \\ \text { capi } & \text { HAB } & \text { dog } & \text { like } & \text { SUBORD } & \text { 3-continue }\end{array}$

'Capi continues to like the dog.' 

$\begin{array}{lllll}\text { (14) } k u & h a & m \tilde{e}=\text { pan }=\varnothing-k r \varepsilon-r & \varnothing-k^{h} \tilde{a} m & m \tilde{e}=\text { pan= }=\varnothing-k r e \\ \text { 1dual } & \text { IRLS } & \mathrm{PL}=1 \mathrm{PL}=\mathrm{R}^{1}-\text { cantar-NMLZ } & \mathrm{R}^{1}-\mathrm{LOC} & \mathrm{PL}=1 \mathrm{PL}=\mathrm{R}^{1} \text {-continuar.AUX } \\ \text { 'Nós vamos continuar cantando.' } & & \end{array}$

(lit. Nós vamos continuar em nosso cantar aqui) (Miranda 2014:274).

(15) wa ha $m \tilde{e}=a=j-i k a j-\varnothing \quad \varnothing-k^{k} \tilde{a} m \quad m \tilde{e}=i=\varnothing-k r \varepsilon$

$1 \mathrm{SG} \quad$ IRLS $\quad \mathrm{PL}=2 \mathrm{SG}=\mathrm{R}^{1}$-esperar-NMLZ $\quad \mathrm{R}^{1}-\mathrm{LOC} \quad \mathrm{PL}=1 \mathrm{SG}=\mathrm{R}^{1}$-continuar.AUX 'Nós vamos continuar esperando vocês.'

(lit. Nós vamos continuar em nossa espera de vocês) (Miranda 2014:274).

As orações com o verbo - kre comportam-se de modo distinto com respeito à concordância com o sujeito. Apesar de haver outros casos similares, em línguas Jê, essa não parece ser uma condição obrigatória quando verbos auxiliares são examinados em uma perspectiva mais ampla.

Já as orações no aspecto habitual são marcadas pelo auxiliar - $p a$ 'ficar', sendo, em Krahô, derivado etimologicamente do verbo 'morar', e pelo verbo $p a$ em Kĩsêdjê (Suyá), o qual foi interpretado por Santos (1997: 89) como um verbo posicional plural, em oposição a forma singular mbra, sem que o autor mencione, contudo, o seu conteúdo semântico. Embora ambas as línguas adotem o mesmo verbo auxiliar com função análoga, as construções das quais ele é núcleo sintático divergem com respeito à concordância do sujeito com verbo auxiliar e à escolha das posposições: -to 'instrumental', em Krahô, exemplo (16) e (17), e kãm 'locativo’ em Kĩsêdjê, exemplos (18) e (19).

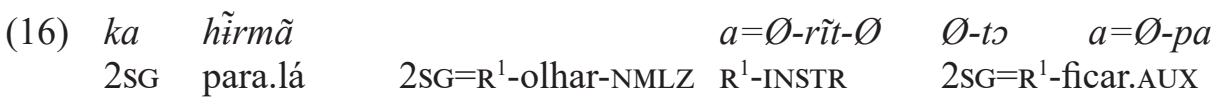
'Você fica olhando para lá.' (Lit. Você fica com o teu olhar para lá) (Miran da 2014:276)

(17) wa $k^{h} w a r \quad \varnothing-k u-r \quad \varnothing-t o \quad i=\varnothing-p a$ 1SG mandioca $\mathrm{R}^{1}$-comer-NMLZ $\mathrm{R}^{1}$-INSTR $1 \mathrm{SG}=\mathrm{R}^{1}$-ficar.AUX

'Nós ficamos comendo mandioca (lit. Nós ficamos com o comer da man dioca) (Miranda 2014:277)

(18) katircye ra aykrokatsi kãm na pa criança MS BRINCAR LOC ? permanecer 'Crianças brincam sempre.' (Santos 1997:88)

(19) karolina ra kuken ro pa N.PESS MS LAVAR INSTR permanecer

'A Karolina está lavando roupa.'(Santos 1997:89)

Perífrases verbais denotando aspecto terminativo são constituídas pelos verbos auxiliares -ikuw 'parar' e -amrẽ(-r) 'acabar'. Os dados (20) e (21), que ilustram o uso desses verbos auxiliares, são provenientes da língua 
Krahô, em que os núcleos lexicais dessas construções, semelhantes àqueles no aspecto progressivo e habitual, são determinados também pela posposição -to 'instrumental'.
ke ha apkhrajre
$m \tilde{e}=h-\jmath ? k u ? k h r \varepsilon-n$
$\varnothing$-to h-ikuw
ENF IRLS criança.PL
$\mathrm{PL}=\mathrm{R}^{2}$-correr.PL-NMLZ
$\mathrm{R}^{1}$-INSTR $\mathrm{R}^{2}$-parar.aux

'As crianças vão parar de correr.'

(lit. As crianças vão parar com o correr delas) (Miranda 2014:282)
$(21)$
wa ha ra $i=j-$-ope- $n$
$1 \mathrm{SG}=\mathrm{R}^{1}$-comer-NMLZ
$\varnothing-t 5$
h-amrẽ
1SG IRLS JÁ
$\mathrm{R}^{1}$-INSTR
$\mathrm{R}^{2}$-acabar.AUX
'Eu vou acabar de comer.'
(lit. Eu vou acabar parar com o meu comer) (Miranda 2014:283)

As orações no aspecto completivo selecionam como auxiliar o verbo $p a$, em Apinajé e Mẽbêngôkre, $-p a(-r)$ em Krahô ${ }^{12}$ e $h w a$ em Kĩsêdjê (Suyá), sendo o padrão sintático bastante regular entre as línguas Jê setentrionais. No entanto, as construções oracionais às quais esse auxiliar se vincula diferem daquelas discutidas acima por não empregarem posposições na qualidade de determinantes dos núcleos lexicais, como pode ser visto a partir dos exemplos abaixo.

\section{Apinajé}

$\begin{array}{llll}n & w a & k a w r y & p a \\ \text { CNJ } & \text { DU } & \text { COLLECT } & \text { CNCL }\end{array}$

'Then we finished gathering.'(Oliveira 2005:336)

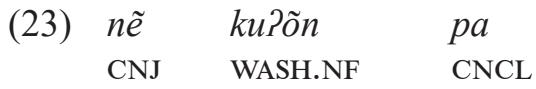

'Then you finished washing.'(Oliveira 2005:336)

\section{Mẽbêngôkre}

aròp nẽ $b a$ i-jàpênh $\quad p a$

já RLS 1SG 1sG-trabalhar COMPL

'Eu já terminei meu trabalho' (Stout e Thomson 1974:3)

$\begin{array}{lllllll}g a & n a & g a & a=j-\tilde{o} & \varnothing-k w \partial & \emptyset-k r \tilde{e}-n & p a \\ 2 & \text { RLS } & 2 & 2-\mathrm{R}^{1} \text {-pertence } & \mathrm{R}^{1} \text {-PART } & \mathrm{R}^{1} \text {-comer-NMLZ } & \text { CESS } \\ \text { 'você cessou } & \text { o seu comer.' (Costa 2015:307) }\end{array}$

12 Em Krahô, esse verbo pode combinar-se com a palavra $t u$, que denota completude relativa a nomes descritivos, resultando na forma composta $p a r=t u$ (Miranda 2014:284) 


\section{Kĩsêdjê (Suyá)}

$\begin{array}{lllll}h n & w a & n- & k-r & h w a \\ \text { ASP } & 1 & \mathrm{R}^{1} \text {-comida } & \text { comer-NMLz } & \text { COMPL } \\ \text { 'eu comi a comida toda (até o fim)' (Santos } & \text { 1997:91) }\end{array}$

liana $r a$ ay-kwã $\quad s-r \quad h w a \quad n \tilde{t} \quad i-m \tilde{a}$ kere N.PESS MS PL-3+POSP $\mathrm{R}^{2}$-dar-NMLZ COMPL CNJ 1-POSP NEG 'Liana deu tudo para os outros, mas nada para mim' (Santos 1997:91)

\section{Krahô (Timbira)}

$i=\varnothing-t \varepsilon \quad h-\tilde{l} \quad \varnothing-t \supset \quad$ - $\quad$-krij-Ø

$1 \mathrm{SG}=\mathrm{R}^{1}$-OBL $\quad \mathrm{R}^{2}$-carne $\quad \mathrm{R}^{1}$-INSTR $\quad \mathrm{R}^{2}$-cortar.PL-NOMLZ COMPL 'eu cortei a carne por completo.' (lit. Houve o cortar completo da carne por mim) (Miranda 2014:285)

$\begin{array}{llll}i=\varnothing-t \varepsilon & \text { pur } & \varnothing \text {-kare-r } & \text { par } \\ 1 \mathrm{SG}=\mathrm{R}^{1}-\mathrm{OBL} & \text { roça } & \mathrm{R}^{1} \text {-limpar-NMLZ } & \text { COMPL }\end{array}$

'eu limpei a roça por completo.' (lit. Houve o limpar completo da roça por mim) (Miranda 2014:285)

Um fato a ser destacado aqui diz respeito ao verbo $h w a$, da língua Kĩsêdjê (Suyá), e-pa da língua Mẽbêngôkre (Xíkrin), nas quais ele também é empregado como verbo pleno, significando 'matar.PL'. Nesses casos particulares, é provável que historicamente esse verbo significasse algo como 'eliminar', 'exterminar', cuja semântica relacionada com a completude de um evento, tenha favorecido a sua gramaticalização como verbo auxiliar. As demais línguas do ramo setentrional, como Apinajé e Krahô (Timbira), teriam, por sua vez, restringido o uso do verbo - pa/-par(-r) apenas como auxiliar (completivo), tornando-o ainda mais gramaticalizado, e consequentemente teriam substituído a antiga forma - pa/-par(-r) para 'matar' pelo verbo -ĩbč 'matar.PL', 'exterminar', 'executar' (Oliveira 2005:379) e -ipcj 'matar.PL', 'eliminar' (Miranda 2014: 85), com conteúdo semântico análogo.

Conforme foi mostrado, nesta seção, as línguas do Grupo I caracterizamse por apresentar consistentemente o padrão sintático, esboçado em (1), com alguma variação relativa ao uso de posposições distintas para o mesmo valor aspectual. Note-se que, em Krahô, há a presença de concordância do sujeito em alguns verbos auxiliares. Outra particularidade a ser sublinhada é a eliminação gradativa da posposição, como se observa nos exemplos de (22) a (29). Retomo essa questão mais adiante, em que argumento que essa e outras mudanças gramaticais envolvendo verbos auxiliares constituem uma das principais diferenças entre as línguas do Grupo I e II. 


\subsection{Línguas do Grupo II}

O Grupo II reúne as línguas Kaingáng e Laklãnõ (Xokléng), as quais representam os casos mais gramaticalizados de verbos auxiliares dentro da família Jê. Apesar de tais verbos serem obrigatórios em diversos tipos de predicados, tanto verbais quanto nominais, bem como parte deles ter correspondentes em línguas Jê setentrionais, mas com funções distintas, o padrão sintático empregado para fins aspectuais é relativamente mais simplificado em Kaingang e Laklãnõ (Xokléng) se comparado com as línguas do Grupo I.

Diferentemente das línguas do Grupo I, nas línguas Kaingáng e Laklãnõ, de um modo geral, o complemento de verbos auxiliares não é regido por posposição e argumentos não são marcam por meio de pronomes absolutivos. Apesar da ausência de posposição, alguns núcleos lexicais ainda conservam a forma nominalizada (não finita), a qual é derivada pelo nominalizador de nome de ação $-g$, em Kaingáng (Gonçalves 2012:35) ${ }^{13}$, e $-g,-n$ e $-\varnothing$ em Laklãnõ (Gakran 2016:160) ${ }^{14}$.

Em ambas as línguas, o aspecto perfectivo é marcado por meio do verbo auxiliar mũ 'ir.PL' (Gonçalves 2011:104), como mostram os exemplos (30) e (31) do Kaingáng, e (32) e (33) do Laklãnõ (Xokléng).

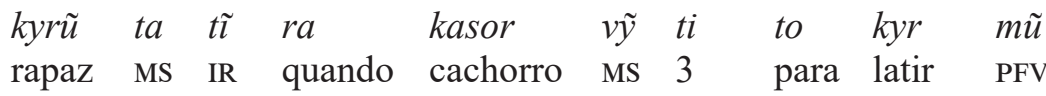

'Quando o rapaz estava passando, o cachorro latiu para ele.' (Gonçalves 2012:222)
(31)
$\begin{array}{lllllll}\text { fongue } & t \tilde{y} & \text { vãnhgénh } & k a r & k a \tilde{t} \tilde{l} & \text { mũ } & k \tilde{a} \\ \text { fongue } & \text { MS } & \text { GUERRA } & \text { depois } & \text { vir } & \text { PFV } & \text { então }\end{array}$
'Depois que o Fongue veio da guerra.' (Gonçalves 2012:222)

13 Ainda que as correspondências entre os morfemas de ambas as línguas sejam evidentes, essa não é a interpretação de Gonçalves (2012:35), segundo a qual esse morfema é acrescido a verbos para expressar "uma referência temporal passada". Por mais que esta seja um efeito secundário decorrente da natureza resultativa da nominalização, impulsionada por mudanças gramaticais, tais formas nominalizadas são parte das exigências estruturais para que um verbo possa funcionar numa função tipicamente nominal ou numa relação de subordinação a outro elemento, afetando a sentença como um todo (Givón 2016).

14 Há, ainda, casos que envolvem o uso de formas nominalizadas obtidas por meio de supleção verbal, como em:
(a)
ti tel wã
he die+stative stative
'He died' (Urban 1985:173)
(b)

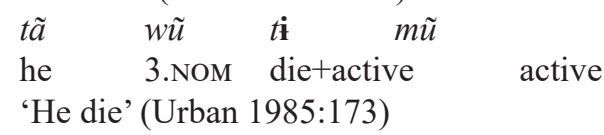


(32) $g o j \quad k i \quad t a \quad k u t a ̃$ mũ água POSP 3MASC cair PERF.AUX

'Ele caiu na água.' (Gakran 2016:171)

(33) jug jé nũ kul kójam mũ pai POSP 1SG roupa comprar PERF.AUX

'Eu comprei roupa para meu pai.' (Gakran 2016:172)

O aspecto imperfectivo nas duas línguas é expresso pelo verbo auxiliar tĩ/tẽ, o qual tem origem no verbo de movimento 'ir.sG'. Em alguns contextos, o verbo auxiliar tĩ/tẽ sugere uma leitura habitual, como nos exemplos (34) e (35) do Kaingáng, e de (36) a (38) do Laklãnõ (Xokléng):
Aranh net $\tilde{y} \quad \tilde{a} n \quad k \tilde{a}$
$k \tilde{a} \quad n \tilde{\imath}-g$ $t \tilde{\imath}$
N.PESS diz.que
LOC:ali
permanece/ficar-PST
$\mathrm{HAB}$
'Áranh disse que morava ali' (Gonçalves 2011:37)

(35) kanhgág si ag ta $k a$ tỹ $p \tilde{\imath}$ han $t \tilde{\imath}$ índio antigo $3 \mathrm{pl} \mathrm{ms}$ pau com fogo fazer hab 'Os antigos sabiam fazer (faziam) fogo com 'pauzinhos' (Gonçalves 2011:120)

(36) jan $m \tilde{a}=m \tilde{e} \quad t \tilde{e}$

cantar 2PL IMPERF.AUX

'Vocês cantam/Vocês vão cantar' (Gakran 2016:186)

(37) kute vĩ $\tilde{e}$ te bantẽ mato ms casa ESPEC POSP IMPERF.AUX

'O mato está (fica) ao redor da casa' (Gakran 2016:194)

(38) ẽnh ja nũ pẽ klég tẽ

1SG ENF 1SG lenha cortar IMPERF.AUX

'Eu vou cortar lenha/Eu mesmo vou cortar lenha.' (Gakran 2016:194)

Em Laklãnõ, diferentemente do Kaingáng, o verbo auxiliar tẽ pode alternar entre uma leitura habitual e prospectiva, como mostram os exemplos (36) e (38). Ainda que a função desse verbo auxiliar difira daquela encontrada em Krahô (aspecto ingressivo), ambos os casos denotam eventos imperfectivos, indicando, dessa forma, a manutenção de certas propriedades semânticas da fonte diacrônica a partir da qual se desenvolveram.

Já para o aspecto progressivo, existem dois modos principais de exprimi-lo nas línguas Kaingang e Laklãnõ (Xokléng): (i) por meio do auxiliar estativo vãal $v \tilde{e}^{15}$, e (ii) por meio de verbos auxiliares posicionais.

15 A função primária desse auxiliar em Kaingang é análoga àquela encontrada em Laklãnõ (Xokléng), sugerindo que ambas sejam formas cognatas. Por esse motivo, considera-se, 
A seleção do auxiliar estativo vã desencadeia uma série de mudanças morfossintáticas, em Laklãnõ (Xokléng), em virtude da natureza nominal dos predicados que o têm como núcleo sintático. Essas mudanças envolvem a marcação de $\mathrm{S}$ e $\mathrm{O}$, que são tratados de modo idêntico, em oposição a A que é regido pela posposição translativa/instrumental tõ, portanto em numa função oblíqua. Nesse aspecto, o núcleo lexical ocorre em sua forma nominalizada (nome de ação). Mudanças no sistema de marcação de caso não são encontradas, por exemplo, em Kaingang, constituindo um enigma a ser resolvido ainda. Do ponto de vista histórico, a fonte diacrônica da qual o auxiliar vãave se desenvolveu não é tão evidente como os demais casos, conforme pode ser visto nos exemplos (39) e (40) do Kaingang e (41) e (42) do Laklãnõ (Xokléng).

$\begin{array}{lllll}k \tilde{a} r u & t a & r e & k o & v \tilde{e} \\ \text { cavalo } & \text { MS } & \text { GRAMA } & \text { comer } & \text { ESTAT }\end{array}$

'O cavalo está comendo (grama).' (Goncalves 2007:133)

(40) $f i$

kajika han ve paçoca

3.FEM canjica fazer ESTAT paçoca

'Ela está fazendo canjica e paçoca.' (Goncalves 2007:133)

(41) ẽnh ja jan-Ø vã

$1 \mathrm{SG}$ ENF cantar-NMLZ ESTAT.AUX

'Eu mesmo que estou cantando.' (Gakran 2016:187)

$\begin{array}{llllll}z i & h a & \text { to } & k o & k a n-\varnothing & v \tilde{a} \\ \text { 3FEM } & \text { ENF } & \text { TRANS } & \text { terra } & \text { cavar-NMLZ } & \text { ESTAT.AUX } \\ \text { 'Ela está cavando buraco.' (Gakran 2016:187) } & \end{array}$

Além do auxiliar estativo $v \tilde{a} / v \tilde{e}$, orações no aspecto progressivo nas línguas Jê meridionais podem ter como núcleo sintático verbos posicionais. As orações marcadas por esses verbos são pragmaticamente orientadas, já que a sua seleção depende de traços semânticos do evento e dos referentes envolvidos nele. Analiso, inicialmente, os dados da língua Laklãnõ (Xokléng), apresentados em seguida:

$\begin{array}{llll}\text { klángne } & \text { nu } & \text { gla } & \text { ne } \\ \text { carne } & \text { MS } & \text { ASSAR } & \text { estar.sentado.AUX } \\ \text { 'A carne está assando (sentada).' (Gakran 2016:178) }\end{array}$

neste estudo, vẽ como auxiliar estativo ao contrário do que foi proposto por Gonçalves (2011:156) que o interpretou como "um operador que exprime Modalidade Epistêmica", embora não seja descartada a hipótese de que sejam palavras homófonas, com funções gramaticais distintas. 


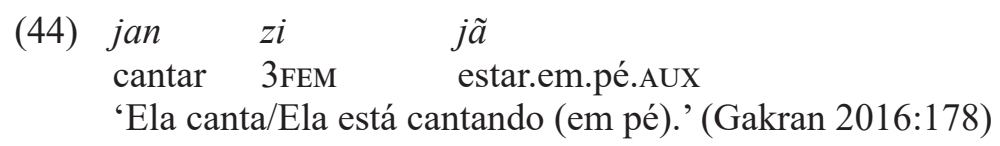

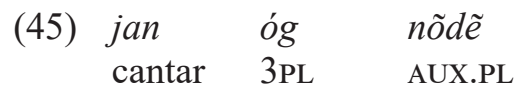

'Eles cantam/Eles estão cantando (em posição indefinida) (Gakran 2016:179)

As línguas Jê setentrionais não marcam a concordância de número entre o sujeito da oração e verbo auxiliar por meio da indexação de pronomes pessoais (dependentes), como ocorre com as línguas do Grupo I. Em Laklãnõ (Xokléng), é o uso de formas verbais supletivas (singular vs. plural) que cumpre esse papel, como em (44) e (45).

A língua Kaingang também emprega verbos posicionais com funções semelhantes àquelas do Laklãnõ (Xokléng), mas difere desta por apresentar usos ainda mais gramaticalizados com respeito a esses verbos, tendo expandido sua função para outros domínios da gramática além de aspecto, como modalidade epistêmica. Nessa língua, é possível empregar dois verbos posicionais em sequência, sendo que o último desempenha o papel de auxiliar, como destacam os exemplos de (46) a (49).

(46) maria tĩ vẽnhkype jẽ

N.PESS MS TOMAR.BANHO em.pé.AUX

'Maria está tomando banho.' (Gonçalves 2011:131)

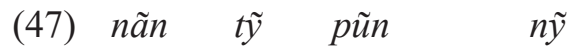

mato MS QUEIMAR deitado/horizontal.AUX

'o mato está queimando.' (Gonçalves 2011:131)

(48) fi ty horta han jẽ nĩ

3SG.F MS HORTA fazer em.pé IMPERF.AUX

'ela está fazendo horta.' (Gonçalves 2011:132)

(49) is y in han je nĩ

1SG.MS CASA FAZER em.pé IMPERF.AUX

'eu estou construindo (levantando) a casa.' (Gonçalves 2011:132)

Conforme observado por Gonçalves (2011:132), os enunciados (48) e (49) poderiam ocorrem sem o verbo posicional jẽ 'estar.em.pé', mantendo sua gramaticalidade. No entanto, a presença apenas do verbo auxiliar (imperfectivo) nĩ exclui qualquer referência à posição, mesmo que ele tenha se desenvolvido de um verbo posicional, significando 'estar.sentado'. Em contraste com verbos auxiliares originários de verbos posicionais, $n \tilde{\imath}$ é o que parece estar mais gramaticalizado em Kaingang, expressando atualmente a noção de duratividade 
do evento (2011:135), como no exemplo (50).

$\begin{array}{lllll}\text { gĩr } & \text { vỹ } & \text { kanhir } & \text { nĩ } & \text { nĩ } \\ \text { criança } & \text { MS } & \text { BRINCAR } & \text { sentado } & \text { IMPERF.AUX } \\ \text { 'A criança } & \text { está brincando.' (Gonçalves } & \text { 2011:134) }\end{array}$

Examino, aqui, a constituição morfossintática das construções oracionais que empregam distintos verbos auxiliares na expressão de aspecto. Embora parte deles corresponda a formas cognatas, como verbos de movimento e posicionais, as funções e os valores aspectuais que expressam diferem em graus variados dentro da família, refletindo diferentes estágios de gramaticalização na história das línguas. Mesmo considerando a variação sincrônica existente, os verbos auxiliares ainda conservam traços semânticos tanto das fontes diacrônicas por meio das quais se desenvolveram quanto das categorias aspectuais às quais se vinculam.

Na seção seguinte, apresento uma proposta de reconstrução sintática do padrão oracional que teria dado origem às construções atuais com verbos auxiliares nas línguas Jê. Questões relativas ao sistema de marcação de caso serão também exploradas, visto que a distribuição e expressão dos argumentos nucleares implicam o uso de formas pronominais distintas, assim como o acionamento de padrões de marcação de caso específicos.

\section{Uma proposta de reconstrução da morfossintaxe de aspecto na família Jê}

A reconstrução de padrões sintáticos tem sido continuamente discutida no âmbito da Linguística História como uma área que ainda oferece grandes desafios teóricos e metodológicos, se comparada com áreas bem estabelecidas, como a reconstrução lexical e fonológica por meio do Método Comparativo (Harris e Campbell 1995; Harris 2003, 2008; Viti 2015). No entanto, em anos recentes, alguns esforços têm sido empreendidos no desenvolvimento de metodologias alternativas para a reconstrução sintática de estágios históricos das línguas ou das fontes etimológicas a partir das quais novos padrões oracionais surgem e se desenvolvem ao longo do tempo (Seržant 2015).

Uma dessas alternativas metodológicas é teoria da Gramaticalização, na medida em que objetiva descrever o "modo por meio do qual formas gramaticais surgem e se desenvolvem através do espaço e tempo" e, ao mesmo tempo, "explicar por que elas são estruturadas no modo que são". Assim, a gramaticalização é concebida como "um processo que é hipotetizado ser essencialmente unidirecional" (Heine 2003:575). Apesar do modelo enfatizar o surgimento de novas formas gramaticais, as quais se desenvolvem a partir de itens lexicais ou tornam ainda mais gramaticais aquelas já existentes na língua, é de utilidade na abordagem das construções oracionais das quais tais formas 
gramaticais pertencem ou mesmo com segmentos discursivos maiores (Heine e Kuteva 2002:2).

A metodologia de reconstrução proposta por Heine (2018) fundamenta-se principalmente em três componentes: (a) reconstrução diacrônica via método comparativo, (b) reconstrução interna, e (c) generalização tipológica. Com base nos dados das línguas Jê apresentados aqui, essa metodologia pode ser exemplificada da seguinte forma. Já que apenas os verbos de movimento e posicionais apresentam correspondências sistemáticas de forma e função, a probabilidade é que essas sejam as formas verbais mais antigas dentro da família, sendo a maioria delas reconstruídas para o Proto-Jê com suas respectivas formas

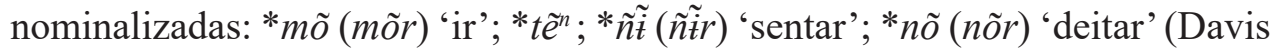
1966:21-22, 1968:43). A reconstrução interna indica que a função lexical seja mais antiga que a auxiliar e não o inverso, já que algumas formas ainda são usadas lexicalmente como verbos plenos, ao passo que outras tenham perdido parcial ou complemente o conteúdo semântico referencial, como o auxiliar estativo vã/vẽ nas línguas Laklãnõ (Xokléng) e Kaingang, ou ainda estejam em processo de desemantização, como o verbo nĩ 'estar.sentado' na última língua. A generalização tipológica nos mostra, por sua vez, que o recrutamento de certos verbos para cumprir funções gramaticais, como tempo, modo e aspecto etc., é uma tendência universal nas línguas e independe da filiação genética. A gramaticalização de verbos auxiliares representa apenas um estágio histórico a partir do qual pode levar, em alguns casos, ao desenvolvimento de morfemas presos (Givón 2001).

Tomando como base o padrão sintático esboçado em (1) e o comportamento morfossintático dos elementos envolvidos nas diversas línguas Jê, proponho uma reconstrução sintática desse padrão em três estágios com mudanças graduais na sua constituição interna.

\section{Estágio I: Proto-Jê}

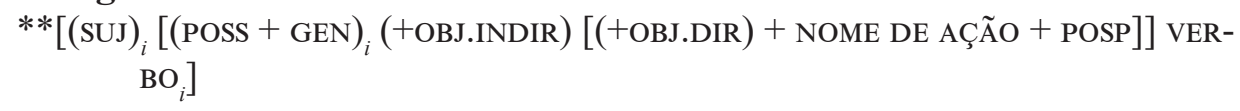

\section{Estágio II}

*[(SUJ $)_{i}\left(+\right.$ OBJ.INDIR) $[(+$ OBJ.DIR $)$ ARG.ABS $=$ =NOME DE AÇÃO + POSP $]$ AUX $\left.{ }_{i}\right]$

\section{Estágio III}

a. *SUJ.OBL (+OBJ.INDIR $)[(+$ OBJ.DIR $)+$ NOME DE AÇÃO $]$ AUX

b. *[(SUJ) (+OBJ.INDIR) (+OBJ.DIR) + NOME DE AÇÃO + AUX $]$

Inicialmente, no Estágio I, as orações apresentavam sujeito próprio e verbos na função de núcleo sintático com eventuais complementos circunstanciais, regidos por posposição. Nesse estágio, a mudança substancial foi o emprego de 
nomes de ação como complemento desses sintagmas, convertendo a estrutura argumental de um verbo para a morfossintaxe nominal via nominalização, cujo argumento de núcleos transitivos passa então a ser codificado em um sintagma oblíquo marcado no caso genitivo. O recrutamento específico de nomes de ação pode ser explicado tanto por fatores funcionais quanto estruturais. Do ponto de vista funcional, nomes de ação conservam as propriedades eventivas do verbo a partir do qual derivam, mas dentro de um protótipo nominal, passando a adquirir, nesse caso, propriedades referenciais e morfossintáticas nominais, como a habilidade de flexionar para gênero, número, caso etc., e funcionar como argumentos de predicados (Givón 2016). O fator estrutural decorre da relação de dependência que é estabelecida com a posposição, já que na qualidade de nome pode ocorrer tanto como argumento de predicado quanto complemento de adjuntos.

Esses fatos explicam, por exemplo, a manutenção da nominalização do núcleo lexical nas línguas do Grupo I, enquanto nas línguas do Grupo II existem apenas alguns vestígios desse estágio, o que nos leva a hipotetizar que a nominalização oracional tenha sido sobreposta por outros processos regulares nessas línguas, como supleção e reduplicação verbais, verbos classificatórios, causativização, dentre outros. Além disso, explica também a marcação pessoal do núcleo lexical ser idêntica à do possuidor de sintagmas possessivos.

O Estágio II abrange a situação encontrada atualmente somente nas línguas do Grupo I. A principal mudança verificada é o apagamento do sintagma oblíquo na função de possuidor de nomes de ação a partir de temas transitivos, mantendo apenas o sujeito nominativo da oração principal com o qual é correferente. No entanto, o argumento absolutivo de nomes de ação derivados a partir de temas intransitivos e transitivos, se pronominais, são marcados pela mesma série de pronomes pessoais e presos no complemento do sintagma posposicional. Esse sistema de marcação de caso ainda é mantido nas línguas em questão. Em Krahô, certos verbos auxiliares, como - $k r e$ 'continuar' e $-p a$ 'ficar', desencadeiam concordância com o sujeito independente da transitividade do núcleo lexical, uma situação que não é observada nas demais línguas Jê setentrionais.

O Estágio III representa, finalmente, o cenário mais gramaticalizado das construções que empregam verbos auxiliares como principal estratégia gramatical para expressão de aspecto. Nesse estágio, coexistem dois padrões oracionais que se manifestam distintamente conforme a categoria aspectual do verbo auxiliar selecionado ou da natureza perfectiva do evento denotado pela construção. A princípio, essa é a situação recorrente nas línguas do Grupo II, cujas mudanças gramaticais resultaram na (i) eliminação da posposição, da qual o núcleo lexical é complemento, (ii) ausência da marcação possessiva no nome de ação, e (iii) cisão na expressão e marcação do argumento sujeito. Em Laklãnõ (Xokléng), por exemplo, o padrão (a) é restrito ao aspecto estativo, em que o argumento sujeito é marcado numa função oblíqua pela posposição translativa/ 
instrumental tõ, enquanto as demais categorias aspectuais (perfectivas e imperfectivas) seguem o padrão (b). Conforme menciono, nas línguas do Grupo II, a concordância entre o sujeito e o verbo auxiliar é estabelecida por meio de formas supletivas e pode ser vista como uma consequência de reajustes funcionais decorrentes de processos de mudança sintática que, por sua vez, desencadearam o desenvolvimento de novos padrões oracionais nessas línguas.

Por mais que as diferenças morfossintáticas sejam notáveis entre as línguas dos dois grupos com respeito às orações com verbos auxiliares, contudo, a comparação dos dados sugere a regularidade de mudanças linguísticas e a possibilidade de que essa possa ter sido a direção natural pelas quais as línguas Jê passaram, sobretudo as do ramo meridional, Kaingang e Laklãnõ (Xokléng). Nas línguas Jê setentrionais, esse cenário pode ser visualizado nas construções que empregam o verbo auxiliar - pa para expressar aspecto completivo, em que os núcleos lexicais não são determinados por posposição, similar às línguas Jê meridionais, conforme evidenciado nos exemplos de (22) a (29). Outros casos, como o verbo -ikuw 'parar', nas variedades Timbira, Krahô (Miranda 2014) e Canela-Ramkokamekrá (Popjes e Popjejs 1986), a variação existente reside no contraste entre eventos perfectivos $v s$. imperfectivos, e que se reflete diretamente na relação de dependência que o nome de ação estabelece com a posposição -to, exemplos (51) e (52), em comparação com (53).

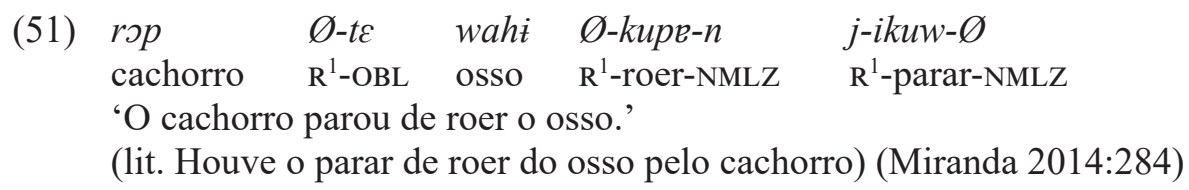

(52) cu-te ih-cakôc jicu

3-PAST 3-speak stop

'He stoped speaking' (Popjes e Popjes 1986:183)

$\begin{array}{lllllll}k u & h a & m \tilde{e} & \text { krero } & \varnothing \text {-kre- } & \varnothing \text {-to } & \text { h-ikuw } \\ \text { 1DU } & \text { IRLS } & \text { PL } & \text { inhame } & \mathrm{R}^{1} \text {-plantar-NMLZ } & \mathrm{R}^{1} \text {-INSTR } & \mathrm{R}^{1} \text {-parar }\end{array}$

'Nós vamos parar de plantar o inhame.'

lit. Nós vamos parar com o plantar inhame (Miranda 2014:282)

A língua Kĩsêdjê (Suyá) apresenta uma situação análoga a do Krahô com respeito à variação relativa à presença/ausência de posposição. Além do verbo $m \tilde{o}$, orações no aspecto progressivo podem também ser marcadas com o verbo auxiliar $t$, ainda que essas sejam menos frequentes na língua. Em (55), o núcleo lexical não é determinado pela posposição $r$, como seria esperado, embora sejam conservadas as demais propriedadesmorfossintáticas, como a nominalização e marcação possessiva. 
$\begin{array}{llll}\text { (55) } h n & w a & a d z=n \tilde{t}-r \tilde{t} x^{\prime} & t \\ \text { ASP } & 1 \mathrm{PL} & 1 \mathrm{PL}=\text { sentar-NMLZ } & \text { ir.AUX }\end{array}$

'Nós estamos sentando.' (Santos 1997:88)

Os casos analisados neste artigo são ilustrativos de como podemos avançar e compreender os mecanismos atuantes em processos de mudança linguística, bem como indicam as prováveis direções que ela pode seguir a partir da reconstrução de padrões sintáticos mais antigos e das fontes diacrônicas a partir das quais se desenvolveram e se gramaticalizaram. Na seção seguinte, formulo algumas considerações gerais e as perspectivas de futuros estudos no âmbito da Linguística Histórica das línguas Jê.

\section{Considerações finais}

Ao longo deste estudo, demonstrei a regularidade de padrões sintáticos em diferentes línguas Jê que empregam verbos auxiliares para exprimir diferentes valores aspectuais, e examinei a constituição morfossintática das construções oracionais usadas para essa função.

Considerando a variação sincrônica existente nesse domínio da gramática das línguas em questão, reuni os membros em dois grupos principiais, a fim de examinar detalhadamente as propriedades morfossintáticas compartilhadas por elas, comparando-as, e de identificar as correspondências de forma e função e eventuais divergências e/ou inovações linguísticas. Os resultados revelam que dos verbos auxiliares, apenas verbos de movimento e posicionais são reconstruídos para estágios históricos mais antigos da família Jê (Cabral et al. 2018), e que os demais verbos auxiliares constituem desenvolvimentos relativamente recentes na história dessas línguas, principalmente daquelas pertencentes ao ramo setentrional (Grupo I). As línguas Jê meridionais (Grupo II), por sua vez, apresentam os casos mais gramaticalizados da família, ainda que sejam percebidas variações consideráveis entre elas, sobretudo quanto ao papel sincrônico de antigas nominalizações e ao sistema cindido de marcação caso existente em Laklãnõ (Xokléng), mas não em Kaingang.

A reconstrução sintática aqui proposta postula a existência de três estágios, a partir dos quais os padrões oracionais que fazem uso de auxiliares se desenvolveram nas línguas Jê, com ênfase nas principais mudanças gramaticais que teriam ocorrido ao longo do desenvolvimento histórico desses padrões. Apesar da escassez de dados abrangendo diversos contornos aspectuais, o presente estudo se constitui em um avanço considerável na testagem de novas hipóteses e na aplicação e avaliação de metodologias alternativas para o estudo histórico-comparativo das línguas Jê.

As construções oracionais com verbos auxiliares nas línguas desse agrupamento genético constituem um excelente campo para a descrição não só dos princípios gramaticais que regem seu uso sincrônico, mas também das 
motivações que levaram ao desenvolvimento dos padrões atuais das línguas, além de nos oferecer ferramentas indispensáveis para a compreensão e explicação de como as experiências sociocognitivas dos falantes e dos contextos discursivopragmáticos podem contribuir na moldagem dos usos linguísticos.

\section{Referências}

Anderson, G. D. S. 2006. Auxiliary verb constructions. New York: Oxford University Press.

Cabral, A. S. A. C et al. 2018. Verbos posicionais em línguas da família Jê (tronco Macro-Jê). Revista Polifonia, v. 25, n. 38.1, p. 01-192.

Cabral, A. S. A. C. Costa, L. S da. 2004. Notas sobre ergatividade em Xikrín. LIAMES, n. 4, pp. 7-19.

Comrie, B. 1976. The syntax of action nominals: a cross-language study. Lingua, vol. 40, n. $2 / 3$.

Costa, L. S. da. 2015. Uma descrição gramatical da língua Xikrín do Cateté (família Jê, tronco Macro-Jê). Tese de Doutorado. Universidade de Brasília: Brasília.

Costa, L. S. da. 2003. Flexão relacional, marcas pessoais e tipos de predicados em Xikrín: Contribuição para os estudos sobre ergatividade em línguas Jê. Dissertação Mestrado, Universidade Federal do Pará.

Cotrim, Rodrigo G. P. M. 2016. Uma descrição da morfologia e de aspectos morfossintáticos da língua Akwê-Xerente (Jê Central). Tese de Doutorado. Universidade de Brasília.

Davis, I. 1968. Some Macro-Jê Relationships. International Journal of American Linguistics, vol. 34, $\mathrm{n}^{\circ}$. 1, p. 42-47.

Davis, I. 1966. Comparative Jê phonology. Estudos Linguísticos: Revista Brasileira de Lingüística Teórica e Aplicada, vol. 1, n. 2, p. 10-24.

Gakran, N. 2015. Elementos fundamentais da gramática Laklãnõ. Tese de Doutorado. Universidade de Brasília - UnB.

Givón, T. 2016. Nominalization and re-finitization. In: Chamoreau, C. EstradaFernández, Z. (Eds.). Finiteness and nominalization. Amsterdam/Philadelphia: John Benjamins Publishing.

Givón, T. 2001. Syntax: a functional-typological introduction. Amsterdan: John Benjamins Publishing.

Gonçalves, S. A. 2012. Perfectividade no Kaingang Sul (Jê). Revista Estudos Linguísticos, n. 41 (1), p. 220-230.

Gonçalves, S. A. 2011. Tempo, Aspecto e Modo em contextos discursivos no Kaingang Sul (Jê). Tese de Doutorado. Universidade Estadual de Campinas: Campinas - SP.

Gonçalves, S. A. 2007. Aspecto no Kaingang. Dissertação de Mestrado. Universidade de Campinas: Campinas - SP.

Harris, A. C. 2008. Reconstruction in syntax: Reconstruction of patterns. In: Ferraresi, 
G. \& Goldbach, M. (Eds.) Principles of Syntactic Reconstruction. Amsterdam/ Philadelphia: John Benjamins Publishing.

Harris, A. C. 2003. Cross-Linguistic perspectives on syntactic change. In: Joseph, B. D. \& Janda, R. D. (Eds.). The Handbook of Historical Linguistics. Oxford: Blackwell Publishing.

Harris, A. C., \& Campbell, L. 1995. Historical syntax in cross-linguistic perspective. Cambridge: Cambridge University Press.

Harris, M. \& Ramat, P. (Eds). Historical development of auxiliaries. Berlin/New York/ Amsterdam: Mouton de Gruyter, 1987.

Heine, B. 2018. Grammaticalization in Africa: Two contrasting hypotheses. In: Heine, B. \& Narrog, H. (Eds.). Grammaticalization from typological perspective. Oxford: Oxford University Press.

Heine, B. 2003. Grammaticalization. In: Joseph, B. D. \& Janda, R. D. (Eds.). The Handbook of Historical Linguistics. Oxford: Blackwell Publishing.

Heine, B. Auxiliaries - Cognitive forces and grammaticalization. New York/Oxford: Oxford University Press, 1993.

Heine, B. \& Kuteva, T. 2007. The genesis of grammar - A reconstruction. Oxford: Oxford University Press.

Heine, B. 2002. World lexicon of grammaticalization. Cambridge: Cambridge University Press.

Melatti, J. C. O mito de Sol e Lua: um comentário. Revista Brasileira de Linguística Antropológica. v. 2, n. 1, 2010, p. 13-76.

Miranda, M. G. no prelo. Verbos auxiliares em Krahô. In. Silveira, M. (Org). Línguas e culturas Macro-Jê. Londrina: EDUEL.

Miranda, M. G. 2014. Morfologia e morfossintaxe da língua Krahô (família Jê). (Tese de Doutorado). Universidade de Brasília.

Newman, J. 2002. A cross-linguistic overview of the posture verbs 'sit', 'stand', and 'lie'. In: Newman, John (ed). The linguistics of sitting, standing, and lying. Amsterdam/Philadelphia: John Benjamins Publishing Company.

Oliveira, C. C. de. 2005. The language of the Apinajé people of central Brazil. (Tese de Doutorado). University of Oregon.

Payne, T. 1997. Describing morphosyntax: A guide for field linguists. Cambridge: Cambridge University Press.

Popjes, J. Popjes, J. 1986. Canela-Krahô. In: Derbyshire, Desmond C. Pullum, Geoffrey K. (eds.) Handbook of Amazonian Languages, vol. 1. Berlin: Mouton de Gruyter.

Rodrigues, Aryon Dall'Igna. 1999. Macro-Jê. In: Dixon, R. M. W. Aikhenvald. Alexandra Y. (Orgs.). The Amazonian languages. Cambridge: Cambridge University Press.

Santos, L. C. dos. 1997. Descrição de aspectos morfossintáticos da língua Suyá/Kisêdjê (Jê). Tese de Doutorado. Universidade Federal de Santa Catarina: Florianópolis. 
Seržant, I. A. An approach to syntactic reconstruction. In: Viti, C. (Ed.). Perspectives on Historical Syntax. Amsterdam/Philadelphia: John Benjamins Publishing.

Stout, M. Thomson, R. 1974. Modalidade em Kayapó. Série Linguística, n 3, p. 69-97.

Urban, G. 1985. Ergativity and accusativity in Shokleng (Gê). International Journal of American Linguistics, vol. 51, $\mathrm{n}^{\mathrm{o}}$. 2, p. 164-187.

Viti, C. 2015. Historical syntax: problems, materials, methods, hypotheses. In: Viti, C. (Ed.). Perspectives on Historical Syntax. Amsterdam/Philadelphia: John Benjamins Publishing.

Watkins, L. J. 1976. Position in grammar: sit, stand, lie. Kansas Working Papers in Linguistics. vol. 1. 